\title{
Association between Lung Ultrasound B-lines and Exercise-Induced Pulmonary Hypertension in Patients with Connective Tissue Disease
}

Kazuki Kagami $^{1}$, Tomonari Harada ${ }^{2}$, Koichi Yamaguchi ${ }^{2}$, Shunichi Kouno ${ }^{2}$, Takahiro Ikoma $^{3}$, Kuniko Yoshida ${ }^{2}$, Toshimitsu Kato ${ }^{2}$, Junichi Tomono ${ }^{2}$, Naoki Wada ${ }^{2}$, Takeshi Adachi $^{1}$, Masahiko Kurabayashi ${ }^{4}$, and Masaru Obokata ${ }^{2}$

${ }^{1}$ National Defense Medical College

${ }^{2}$ Gunma University Graduate School of Medicine School of Medicine Faculty of Medicine ${ }^{3}$ Gunma University Hospital

${ }^{4}$ Gunma University Graduate of Medicine

May 3, 2021

\begin{abstract}
Background: Identification of elevation in pulmonary pressures during exercise may provide prognostic and therapeutic implications in patients with connective tissue disease (CTD). Interstitial lung disease (ILD) is common in CTD patients and subtle interstitial abnormalities detected by lung ultrasound could predict exercise-induced pulmonary hypertension ( $\mathrm{PH}$ ). Methods and Results: Echocardiography and lung ultrasound were performed at rest and bicycle exercise in CTD patients (n=41) and control subjects without CTD $(n=24)$. Ultrasound B-lines were quantified by scanning four intercostal spaces in the right hemithorax. We examined the association between total B-lines at rest and the development of exercise-induced PH during ergometry exercise. Compared to controls, the number of total B-lines at rest was higher in CTD patients $(0[0,0]$ vs. $2[0$, 9], $\mathrm{p}<0.0001$ ) and was correlated with radiological severity of ILD assessed by computed tomography (fibrosis score, $\mathrm{r}=0.70$, $\mathrm{p}<0.0001$ ). Pulmonary artery systolic pressure (PASP) was increased with ergometry exercise in CTD compared to controls ( $48 \pm 14$ vs. $35 \pm 13 \mathrm{mmHg}, \mathrm{p}=0.0006$ ). The number of total B-lines at rest was highly correlated with higher PASP ( $\mathrm{r}=0.52$, $\mathrm{p}<0.0001$ ) and poor right ventricular pulmonary artery coupling (tricuspid annular plane systolic excursion/PASP ratio, $\mathrm{r}=-$ $0.31, \mathrm{p}=0.01$ ) during peak exercise. The number of resting B-lines predicted the development of exercise-induced $\mathrm{PH}$ with an area under the curve $0.79(\mathrm{p}=0.0003)$. Conclusions: These data may suggest the value of a simple resting assessment of lung ultrasound as a potential tool for assessing the risk of exercise-induced PH in CTD patients.
\end{abstract}

\section{Hosted file}

CTD B-line Main.pdf available at https://authorea.com/users/411670/articles/520629association-between-lung-ultrasound-b-lines-and-exercise-induced-pulmonary-hypertensionin-patients-with-connective-tissue-disease 
Figure 1

Total number of B-lines in the 4 lung regions

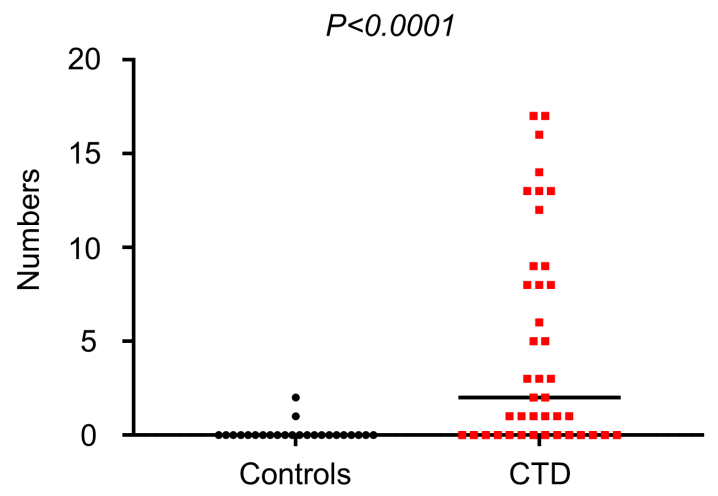

Figure 2

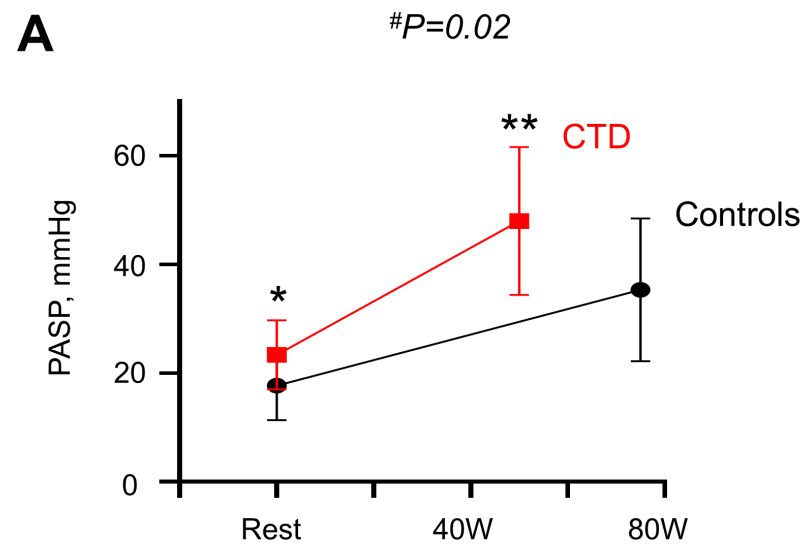


Figure 3

A

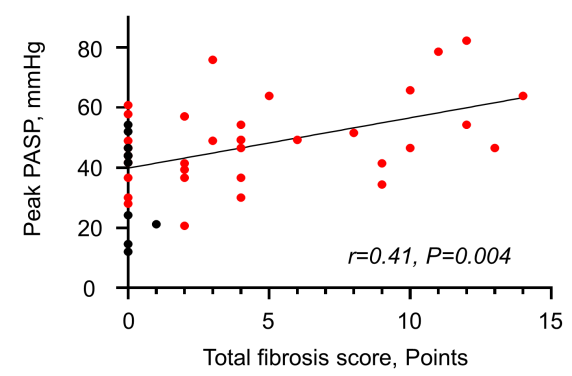

B

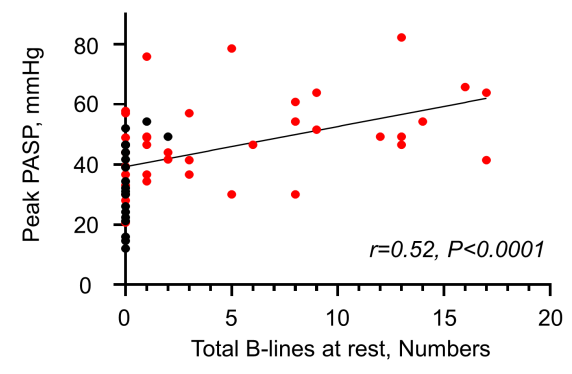

- CTD Controls

Figure 4
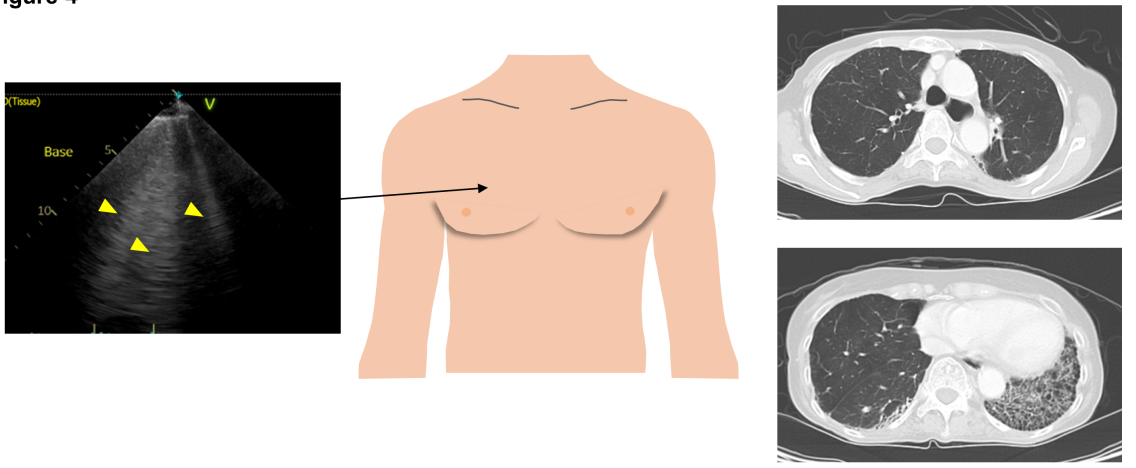

Rest

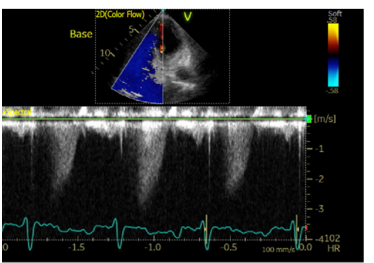

PASP $30 \mathrm{mmHg}$
Peak exercise

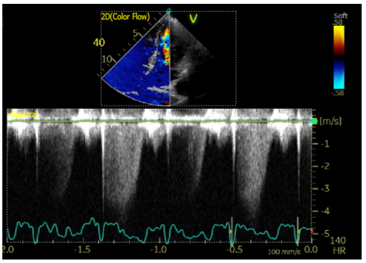

PASP $58 \mathrm{mmHg}$ 